\title{
Physiological microbial exposure has a temporally limited inhibitory effect on lung ILC2 responses to allergens in mice
}

Stephen Jameson ( $\sim$ james024@umn.edu )

University of Minnesota Medical School https://orcid.org/0000-0001-9137-1146

Katharine Block

University of Minnesota Medical School https://orcid.org/0000-0001-9488-3577

Koji lijima

Mayo Clinic Arizona

Mark Pierson

University of Minnesota Medical School

Daniel Walsh

University of Minnesota Medical School

Rinna Tei

Mayo Clinic Arizona

Tamara Kucaba

University of Minnesota Medical School

Julie Xu

University of Minnesota Medical School

Thomas Griffith

University of Minnesota Medical School

Henry McSorley

University of Dundee

Hirohito Kita

Mayo Clinic https://orcid.org/0000-0002-6854-2936

Article

Keywords:

Posted Date: December 17th, 2021

DOI: https://doi.org/10.21203/rs.3.rs-1139168/v1 
License: (c) (i) This work is licensed under a Creative Commons Attribution 4.0 International License. Read Full License

Additional Declarations: There is NO Competing Interest.

Version of Record: A version of this preprint was published at Nature Immunology on November 21st, 2022. See the published version at https://doi.org/10.1038/s41590-022-01350-8. 
4 Katharine E. Block ${ }^{1,2}$, Koji lijima ${ }^{3}$, Mark J. Pierson ${ }^{1,2}$, Daniel A. Walsh ${ }^{1,2}$, Rinna Tei ${ }^{3,4}$, Tamara A.

5 Kucaba $^{5}$, Julie Xu ${ }^{5}$, Thomas S. Griffith ${ }^{2,5}$, Henry J. McSorley ${ }^{6}$, Hirohito Kita3 ${ }^{*}$, Stephen C.

6 Jameson ${ }^{1,2 *}$

7

$8{ }^{1}$ Department of Laboratory Medicine and Pathology, University of Minnesota, Minneapolis, MN

955455, USA

$10 \quad{ }^{2}$ Center for Immunology, University of Minnesota, Minneapolis, MN, USA

$11{ }^{3}$ Division of Allergy, Asthma and Clinical Immunology and Department of Medicine, Mayo Clinic

12 Arizona, Scottsdale

$13{ }^{4}$ Department of Pulmonary Medicine and Clinical Immunology, Dokkyo Medical University,

14 Tochigi

$15{ }^{5}$ Department of Urology, University of Minnesota, Minneapolis, MN, USA

$16{ }^{6}$ Division of Cell signaling and Immunology, School of Life Sciences, University of Dundee,

17 Dundee, United Kingdom

$18 *$ Corresponding authors.

20 Send correspondence to james024@umn.edu (S.C.J.) or kita.hirohito@mayo.edu (H.K.)

21 


\section{Abstract}

23 The impact of microbes on restraining the immune response to allergens has been extensively

24 studied and is a key element of the hygiene hypothesis. Lung type 2 innate lymphoid cell

25 responses to airway allergens can be inhibited by administration of a number of microbial

26 products; however, it is unclear whether such an effect would be observed with natural

27 infections and how sustained any observed inhibitory effects would be. To answer these

28 questions, we used a murine model of physiological microbial exposures through cohousing SPF

29 laboratory mice with pet store mice and examined the acute type 2 response to intranasally

30 delivered fungal allergen extract Alternaria alternata. We found laboratory mice cohoused with

31 pet store mice for two weeks display a suppressed ILC2 response to $A$. alternata which resulted

32 in reduced eosinophilia. By comparison, mice cohoused for at least two months had ILC2 and

33 eosinophil responses similar to SPF mice despite dramatic changes to the composition of the

34 immune cell populations in the lungs. Lung ILC2 in two-month cohoused mice were still

35 sensitive to subsequent inflammatory cues, as administration of poly(I:C) was able to suppress

36 ILC2 activation and eosinophilia equally well in SPF and two-month cohoused animals. These

37 findings suggest ILC2 dynamically respond to their environment and are not easily desensitized

38 long-term. 
Introduction

40 Allergic asthma affects 300 million people globally, with the incidence increasing over the last

41 century $^{1}$. The most common type of allergic asthma is mediated by type 2 immunity,

42 characterized by cytokines interleukin-4 (IL-4), IL-5, and IL-13, and recruitment of eosinophils,

43 mast cells, and other immune cells into the lungs ${ }^{2}$ (Holgate 2012). Historically, CD4 ${ }^{+}$type 2 T

44 helper cells (Th2) have been thought to be the main producers of IL-5 and IL-13 and thus

45 studied as the cellular mediators of allergic asthma. However, since the discovery and

46 characterization of the tissue resident innate lymphoid cells (ILCS) in the past decade, the large

47 contribution of ILC2 in allergic responses has been appreciated ${ }^{3}$. Specifically, lung-resident ILC2

48 rapidly respond to inflammatory signals induced in the lung epithelium, particularly IL-33, by

49 protease-containing allergens $\mathrm{s}^{4,5}$ and are necessary for a maximal adaptive response from Th2

50 against allergens ${ }^{6,7}$.

51

52 The hygiene hypothesis and the related "old friends" hypothesis posit that improved sanitation

53 and hygiene reduce our exposure to pathogens and commensals and this reduced microbial

54 exposure leads to inappropriate responses to allergens ${ }^{8,9}$. Retrospective and prospective

55 studies of children have found compelling links between exposures to microbes and allergic

56 sensitization. For example, having older siblings, starting day-care at an early age, and growing

57 up on a farm are thought to be proxies for increased microbial exposures and all are associated

58 with lower risk to allergic sensitization ${ }^{10,11,12,13,14,15}$. Additional studies have more directly

59 made the connection between microbes and allergies; high endotoxin levels in house dust,

60 increased intestinal microbial diversity, and lack of antibiotic use in infancy are all associated 
61 with lower risk of atopy ${ }^{16,17,18,19}$. While not all studies investigating these links between

62 microbes and allergic responses report significant results ${ }^{20}$, the human studies have motivated

63 basic research into the effects of microbial exposure on the immune response to allergens in

64 animal models, in order to tease apart potential mechanisms for these phenomena. Microbial

65 products or synthetic analogs such as unmethylated CpG DNA, household dust containing LPS,

66 bacterial lysate OM-85, and the double stranded RNA analog poly(I:C) all decrease allergic

67 sensitization in various allergic asthma models $21,22,23,24,25,26$ albeit some studies reported low

68 levels of LPS actually worked as an adjuvant and increased allergic responses in certain allergic

69 models ${ }^{27,28}$. Intranasal administration of the Heligmosomoides polygyrus-derived protein HpARI

70 interferes with ILC2-activating cytokine IL-33 and prevents sensitization to airway allergens ${ }^{29,} 30$.

71 Deliberate infection with a gammaherpesvirus that infects the lungs was also found to be

72 protective in an allergic asthma model ${ }^{31}$. Various microbes and microbial products can interfere

73 with the response of lung ILC2 to allergens suggesting an early block in the nascent type-2

74 immune response. In particular, type I and II interferons induced by acute viral infection or

75 microbial products reduce IL-5 and IL-13 production by ILC2 $25,32,33,34$. Most of these studies

76 focus on the acute effects of infection by individual pathogens or transient treatment with

77 microbial products: the short- and long-term effects of diverse, physiologically acquired

78 infections on the immune response to inhaled allergens has not been assessed.

80 Improved animal models of human diseases such as allergic asthma would be highly useful for

81 clinicians and basic researchers, especially for answering questions related to how immune

82 experience impacts allergic responses. Our group has the unique opportunity to study the 
83 effects of physiological microbial exposure on immune responses in mouse models. As we have

84 previously published, inbred laboratory mice cohoused with a pet store mouse continuously for

85 two months acquire natural infections, resulting in an immune-experienced phenotype ${ }^{35}$.

86 Through this prior work we have demonstrated that the gene signature of peripheral blood

87 mononuclear cells (PBMC) of cohoused mice more closely resembles the gene signature of

88 human PBMC, while SPF mice PBMC more closely resemble neonatal cord blood ${ }^{35}$. We sought

89 to utilize this "dirty" mouse model to test whether microbial exposures from pet store mice

90 could alter the lung ILC2 response to airway allergens at timepoints both early and late in

91 cohousing. Timing of transmission of microbes through cohousing is not instantaneous but

92 happens within the first two weeks of introduction of the pet store mouse. After two months of

93 cohousing infections are generally resolved or in a chronic or latent phase, but signs of

94 continued inflammation are present, such as elevated serum cytokine levels ${ }^{36}$. We therefore

95 tested the innate immune response to $A$. alternata (A/t) after short-term (two-week) cohousing,

96 modeling a response to an allergen in the midst of an immune response to infection, as well as

97 after long-term (at least two-month) cohousing, to model response to allergen in a healthy

98 individual that has prior microbial experience. Short-term cohousing led to a substantial

99 reduction in the ILC2 and eosinophil responses to intranasal Alt administration, in keeping with

100 prior studies investigating the acute effects of deliberate infections and treatment with

101 microbial products. However, diverse microbial experience did not lead to permanent changes

102 in reactivity to inhaled allergens; despite changes in lung immune cell composition and a delay

103 in the response to Alt exposure, ILC2 activation and lung eosinophilia was restored in long-term

104 cohoused mice. Furthermore, the type-2 response of long-term cohoused animals could be 
105 inhibited by acute treatment with poly $(\mathrm{I}: \mathrm{C})$, similar to the inhibition observed in SPF animals.

106 Together, these data indicate lung ILC2 are highly sensitive to recent inflammatory and

107 inhibitory signals in the tissue microenvironment, but prior microbial experience does not lead

108 to sustained reprogramming of the ILC2 response to inhaled allergens. These findings suggest

109 the immune system is "reset" for type-2 responses to allergens following acute control of

110 microbial infections, with implications for developing treatments for allergic diseases. 


\section{Results}

112 Diverse microbial exposure leads to transient impairment of lung ILC2 and eosinophil

113 responses to intranasal $\boldsymbol{A}$. alternata. We utilized an established mouse model, involving

114 intranasal exposure with $A$. alternata extract $(A / t)$, evaluating the innate immune response in

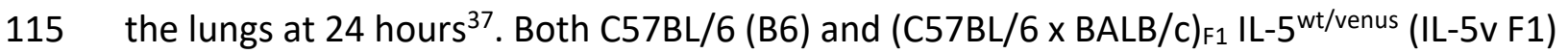

116 animals were used, the latter to serve as a reporter for IL-5 production. Shortly before sacrifice,

117 a fluorescent anti-CD45 antibody was injected intravenously so that cells in the vasculature

$118\left(\mathrm{CD}_{5} \mathrm{iv}^{+}\right)$could be distinguished from cells in the lung parenchyma and airspace (CD45 iv', Fig

119 S1a), providing a more flexible way to analyze lung-resident immune cell populations than, for

120 example, bronchoalveolar lavage. As expected, there were few eosinophils in the lungs of SPF

121 mice given intranasal PBS 24 hours prior, but after Alt treatment this population increased

122 significantly (Fig S1a, b). Previous studies indicate that the type-2 response to allergens can be

123 inhibited by exposure to microbial products ${ }^{25}$. One example is the H. polygyrus protein HpARI,

124 known to bind and interfere with ILC2 activating cytokine IL-33 ${ }^{30}$. Indeed, intranasal

125 administration of HpARI concurrent with Alt exposure led to significantly reduced lung

126 eosinophilia (Fig S1b). Likewise, HpARI inhibited production of IL-5 by lung ILC2 in response to

127 Alt treatment, as revealed by reduced frequency and expression intensity of the IL-5 reporter

128 and reduced surface expression of the IL-33 receptor ST2 and high affinity IL-2 receptor CD25

129 (Fig S1C-f). These results demonstrate ILC2 responses to Alt can be inhibited by microbial

130 products in B6 mice and IL-5v F1 reporter mice. 
132 While those studies confirm acute intranasal exposure to microbial products restrains the ILC2

133 response to Alt, it was unclear whether physiological microbial exposure would have similar

134 effects. To answer this, we cohoused SPF B6, BALB/c, and IL-5v F1 animals with mice purchased

135 from local pet stores. As expected ${ }^{35}$, we observed seroconversion against common murine

136 pathogens and the diversity increased over the cohousing period (two weeks vs two months,

137 Fig S1g-h). We also assayed serum cytokine and chemokine levels over time after cohousing,

138 observing levels of most factors (including TNF- $\alpha$, CXCL10, and IL-6) peaked at approximately

139 10-14 days of cohousing and then plateaued, at elevated levels relative to SPF mice, up to at

140 least two months (Fig 1a, Fig S1i). These findings align with published results showing the

141 frequency of $\mathrm{CD} 44^{\text {high }}$ blood $\mathrm{CD} 8^{+} \mathrm{T}$ cells (indicative of antigen experience) also peaked at

142 approximately two weeks post cohousing ${ }^{35}$.

144 These data suggest the 2-week time point is suitable to assess the short-term effects of recent

145 microbial exposure physiologically transmitted through mouse-to-mouse contact. Similar to the

146 effects of acute treatment with defined microbial products, short-term cohousing led to a

147 significant reduction in the appearance of lung parenchymal eosinophils following Alt exposure;

148 while eosinophil numbers increased $>40$-fold in SPF mice, this was limited to only a $\sim 7$-fold

149 increase in two-week cohoused mice (Fig 1b). The frequency and expression levels of IL-5

150 venus ${ }^{+}$lung ILC2 was also reduced in two-week cohoused mice (Fig 1c-d), while the total

151 numbers of ILC2 were not impacted by cohousing and decreased slightly in number 24 hours

152 after Alt treatment in both groups (Fig S2a), perhaps due to the reported phenomenon of it

153 being more difficult to extract activated lymphocytes from tissues ${ }^{38}$. We considered that 
154 cohousing may have affected IL-5 producing CD4 ${ }^{+} \mathrm{T}$ cells in the lung, but the population of

155 parenchymal CD4 ${ }^{+}$T cells was similarly small in two-week cohoused and SPF animals, and no

156 significant change in the number of IL-5 venus expressing CD4 ${ }^{+}$T cells was induced by

157 cohousing or Alt treatment (Fig S2b-d). These results demonstrate physiological transmission of

158 natural murine pathogens limits ILC2 responses against an airway allergen and reduces lung

159 eosinophilia.

160

161 To determine the extent to which physiological microbial exposure led to a sustained change in

162 the lung response to allergens, we next assessed mice that had been cohoused long-term, for at

163 least two months. In sharp contrast to our findings using short-term cohoused mice, the

164 response to Alt in two-month cohoused animals was indistinguishable from that in SPF animals,

165 inducing similar numbers of parenchymal eosinophils (Fig 1e) and similar production of IL-5

166 venus by lung ILC2 at 24 hours after Alt treatment (Fig1f-g). Together, these data suggest that

167 acute physiological microbial exposure does indeed provoke an impaired type-2 response to an

168 inhaled allergen, but this blunted response is not sustained long-term despite ongoing systemic

169 inflammation above levels observed in SPF mice.

170

171 Delayed initial response to A. alternata in long-term cohoused mice. It was possible the lungs

172 of long-term cohoused mice revert to the characteristics of SPF mice, despite the effects of the

173 cumulative infectious history (Fig S1g) and sustained changes in serum cytokines and

174 chemokines (Fig 1a) ${ }^{35}$, leading to identical responses to intranasal Alt. To assess this possibility,

175 we first examined the cellular composition of the lungs in SPF and long-term co-housed mice. 
176 Multiple immune cell populations were increased in number in cohoused animals (Fig 2a, see

177 Fig S3a-c for gating strategy), and previously published whole lung RNA sequencing revealed

178 that the overall gene expression in the lungs is significantly altered by cohousing ${ }^{39}$. However,

179 the size of the ILC2 population was unchanged and eosinophils only modestly higher in mice

180 cohoused for at least two months (Fig 2a). Lung ILC2 were characterized in more detail and in

181 addition to being equal in number in SPF and two-month cohoused mice, the cells had a similar

182 surface phenotype, including CD44, ST2, CD25, KLRG1, and CD127 (Fig 2b). The transcription

183 factor Gata3 was also similarly expressed in these two groups (Fig 2c).

184

185 We next assessed the response to Alt in SPF and $\geq 2$-month cohoused mice kinetically. One of

186 the first steps in the type 2 response to acute airway allergen is the release of IL-33 from lung

187 cells ${ }^{37,40}$. This alarmin is stored in the nucleus of cells but is rapidly secreted upon allergen

188 sensing ${ }^{41}$. We collected bronchoalveolar lavage fluid (BALF) from the lungs of SPF and two-

189 month cohoused mice one hour after Alt treatment and measured IL-33 by ELISA. IL-33 was

190 lower in Alt treated long-term cohoused mice than SPF mice, while IL-33 levels in BALF were

191 low in all PBS treated control mice (Fig 3a). To determine whether this decreased IL-33 would

192 have an impact in type 2 cytokine levels immediately after allergen treatment, BALF and lung

193 samples were collected 4.5 hours after Alt exposure to measure IL-5 and IL-13 levels by ELISA.

194 Reflecting the lower IL-33, cohoused mice also had reduced IL-5 and IL-13 in the lungs and BALF

195 (although IL-13 in the BALF did not reach statistical significance) (Fig 3b-c and Fig S4a-b). We

196 also measured IL-5 and IL-13 in the lungs of SPF and two-month cohoused BALB/c mice and

197 observed a similarly reduced cytokine response at this acute timepoint in this mouse strain (Fig 
S4c-d). This, along with consistent responses in previous experiments between B6 mice and IL-

$1995 v$ F1 reporter mice, demonstrates the observed phenomena in cohoused mice are not specific

200 to one particular inbred strain of laboratory mice. To test to what extent the lower initial

201 amounts of IL-5 and IL-13 were due entirely to reduced initial IL-33, or if two-month cohoused

202 mouse ILC2 had reduced sensitivity to IL-33, mice were treated intranasally with recombinant

203 IL-33 and IL-5 and IL-13 were measured 4.5 hours later. We found IL-5 was slightly reduced in

204 cohoused mouse lungs, but lung IL-13 and BALF IL-5 and IL-13 were unchanged between SPF

205 and cohoused (Fig 3d-e, Fig S4e-f). These results suggest that lower IL-5 production 4.5 hours

206 after Alt treatment in two-month cohoused mouse lungs is in large part due to reduced IL-33,

207 but may also reflect reduced sensitivity to IL-33 by ILC2 in those animals.

208

209 Together, these findings suggest sustained changes in the immune cell composition of the lungs

210 and altered initial responses toward Alt in long-term cohoused mice. Nevertheless, these

211 alterations evidently do not prevent lung ILC2 activation and efficient eosinophil infiltration 24

212 hours after Alt exposure in two-month cohoused mice (Fig 1). This suggests the type-2 response

213 to allergens is delayed but not inhibited by long-term cohousing with pet store mice.

215 Response to repeated A. alternata exposure is similar in SPF and two-month cohoused mice.

216 It was possible the altered initial response toward Alt in long-term cohoused mice would impact

217 the effects of repeated allergen exposure. To test this, we adapted a model of repeated airway

218 Alt treatment in SPF and two-month cohoused animals. We treated mice intranasally with the

219 allergen every other day three times and analyzed 24 hours after the last dose in order to focus 
on the innate immune response. After repeated exposure to Alt, there was an expansion of ILC2

221 in the lungs of both SPF and two-month cohoused mice (Fig 4a), and a subsequent recruitment

222 and/or expansion of eosinophils, neutrophils, and conventional (Foxp3 ${ }^{-}$) and regulatory (Foxp $3^{+}$)

$223 \mathrm{CD}^{+} \mathrm{T}$ cells in both groups of mice (Fig 4b-e). While most of these cell populations were slightly

224 elevated in PBS-treated two-month cohoused mice compared to PBS-treated SPF mice,

225 reflecting the steady-state changes (Fig 2a), Alt treatment led to cell populations of

226 approximately equal size in SPF and $\geq$ two-month cohoused lungs. The magnitude of the

227 recruited or expanded populations may therefore be slightly decreased in two-month cohoused

228 mice (since the number of cells was higher in these mice before treatment); however, a type 2

229 allergic response clearly was not substantially inhibited in $\geq$ two-month cohoused mice after

230 repeated allergen exposure.

232 The similar responses by SPF and two-month cohoused mice to repeated Alt treatment, as well

233 as at 24-hours following a single treatment, demonstrate a threshold of activation of ILC2 is

234 reached in two-month cohoused mice, prompting a type 2 immune response despite sustained

235 alterations to the immune cell composition of the lungs and serum cytokines and chemokines

236 which accompany long-term cohousing.

238 Acute microbial stimulus is capable of re-suppressing type 2 responses to $A$. alternata in two-

239 month cohoused mice. We established ILC2 responses to Alt could be inhibited by microbes

240 introduced through cohousing two weeks after initiating cohousing with a pet store mouse, but

241 responses at two months of cohousing were similar to SPF mouse responses (Fig 1). Serum 
242 cytokine levels in cohoused mice over time demonstrated that inflammation peaks around 2

243 weeks and is lower by two months, although still elevated over SPF mice (Fig 1a). Certain

244 inflammatory cytokines, such as interferons, inhibit ILC2 responses $25,32,33,34$, and the increased

245 level of inflammation at two weeks may account for the suppression of these cells early but not

246 late after cohousing. On the other hand, it was possible long-term cohoused mice were now

247 resistant to the ability of acute microbial product exposure to impair type-2 responses against

248 allergens. For example, the elevated levels of interferon-induced factors found at steady-state

249 in serum (Fig 1a) $)^{36}$ might lead to sustained desensitization of some responses to interferons.

250 Alternatively, sustained levels of interferons in two-month cohoused mice may be below the

251 threshold to modulate ILC2s. To test these possibilities, we pre-treated SPF and two-month

252 cohoused mice intranasally with poly $(\mathrm{I}: \mathrm{C})$ one day before treating with Alt and examined the

253 response 24 hours after allergen exposure. As previously described ${ }^{25}$, poly(l:C) pretreatment

254 suppressed IL-5 production from ILC2 and eosinophil recruitment into the lungs, and also

255 caused reduced expression of ST2 and CD25 on ILC2, reflecting reduced levels of activation of

256 these cells (Fig 5a-e). Two-month cohoused mice pre-treated with poly(l:C) before Alt

257 treatment similarly had reduced lung eosinophilia (Fig 5a), reduced IL-5 venus expression in

258 ILC2 (Fig 5b-c), and reduced surface expression of ST2 and CD25 on ILC2 (Fig 5d-e),

259 demonstrating the type-2 immune response to allergens in these animals is still sensitive to

260 blockade by acute production of inflammatory factors, such as interferons. 


\section{Discussion}

262 In addition to genetic factors, an individual's history of microbial exposures is an important 263 component that can influence the susceptibility to atopic diseases such as allergic asthma. We

264 used the "dirty mouse" model, physiologically acquired natural murine pathogens and

265 commensals through cohousing laboratory mice with pet store mice, to test the effects of

266 diverse microbial experience on responses to a fungal allergen delivered to the airways. We

267 found short-term cohousing (two weeks) did indeed lead to a suppressed type 2 responses to

268 Alt. However, the ability to mount a type-2 response against single or repeated exposures to

269 the allergen was restored in long-term (at least two months) cohoused mice, despite reduced 270 initial induction of ILC2 activation.

271

272 A central finding from our studies is that prior microbial experience does not permanently 273 reprogram flexibility in immune reactivity: while short-term cohousing resulted in inhibition of

274 this response, this was restored in long-term cohoused mice. These findings imply - despite 275 sustained changes in inflammatory factors in the serum and immune cell composition of the 276 lungs - the immune system can "reset," in this case restoring the ability to mount a type-2

277 immune response to an allergen. Furthermore, despite diverse microbial experience in long-

278 term co-housed mice, they were still responsive to blockade of this type- 2 response by acute 279 exposure to inflammatory cues (modeled by poly(I:C)).

280

281 These findings support underlying concepts of the hygiene/“old friends" hypotheses, but argue

282 it is recent microbial exposures, rather than the cumulative infectious history, which dictates 
283 type-2 responsiveness to allergens. This conclusion has logical appeal, since it would behoove

284 the immune system to remain flexible for appropriate responses independent of previous

285 microbial exposures - for instance, being able to mount a type-2 immune response against

286 helminthic infections despite a history of numerous type-1 responses against intracellular

287 pathogens. Our studies showed physiological, unsynchronized transmission of microbes

288 (including several viruses) causes transient blockade of type-2 responses to allergens but that

289 this effect is largely lost once acute infections are under control. This immunological "resetting"

290 is in contrast to responses to other immune challenges, such as infection by Listeria

291 monocytogenes, Plasmodium berghei, induction of sepsis and influenza vaccination, all of which

292 are sustainably altered in $\geq$ two-month cohoused mice $35,36,39$. Hence, several aspects of the

293 immune response changed long-term following diverse microbial experience, but the ability to

294 mount a type-2 response to inhaled allergens is evidently not one of these.

296 In epidemiological studies, human subjects may have had months, years, or decades of

297 microbial exposures (depending on the age of participants). The identities and timing of the full

298 range of bacteria, viruses, and fungi an individual has been exposed to in their lifetime cannot

299 be determined, but these exposures are believed to be an important component in determining

300 one's susceptibility to developing allergic conditions. The cohoused mouse model we have

301 employed in this study provides the benefits of animal models - genetic homogeneity and

302 genetic tools such as fluorescent gene reporters, short experimental timeframes, and ability to

303 characterize cellular and molecular responses within the tissues - but also allows us to study 
304 the impacts of the physiological microbial exposures on immune responses that cannot be 305 addressed with SPF animals ${ }^{42,43}$.

307 Whether ILC2 would be more, less, or equally responsive to airway allergen in cohoused versus 308 SPF mice was not obvious prior to our studies, as previous publications could support opposing 309 hypotheses. For example, two-month cohoused mice showed evidence of strong type-1 310 immune responses, yet also displayed evidence of type 2 responses, including elevated IL-5, IL-

31113 and IgE levels in the serum, consistent with a history of, or ongoing, parasitic infections ${ }^{35,36}$.

312 Some parasitic infections impede subsequent allergic responses $44,45,46$. Conversely, this type 2

313 signature may have been an indicator of immune cells poised to robustly respond to additional

314 type 2 stimuli such as an airway allergen. In humans, certain severe respiratory infections in

315 infants and children, such as respiratory syncytial virus and rhinovirus, are associated with

316 increased likelihood of wheeze and allergic asthma, potentially through vulnerability of

317 unresolved lung tissue damage ${ }^{47,48}$. We did indeed observe evidence of prior lung infections,

318 with increases of many immune cell populations in the lungs such as neutrophils; however, this

319 did not result in increased sensitivity to allergens. In fact, two-month cohoused mice had

320 reduced IL-33 levels in the BAL after Alt treatment. Other studies have demonstrated type I and

321 II interferons can suppress IL-5 and IL-13 production from lung ILC2 ${ }^{25,33,34}$. IFN- $\gamma$ was elevated

322 over SPF mice in the serum of two-month cohoused mice, and an interferon signaling signature

323 was characterized from PBMC sequencing ${ }^{35,36}$, but the peak in inflammatory cytokines,

324 including IFN- - , was around two weeks after cohousing (Fig 1a), the time at which Alt responses

325 were suppressed. We found acute administration of poly $(I: C)$ before Alt treatment could 
suppress ILC2 responses in both SPF and two-month cohoused mice, suggesting a new spike in

327 interferons could once again suppress ILC2 responses to allergens in microbially-experienced 328 mice.

330 One potential concern about using the cohoused mouse model is the heterogeneity in the

331 timing and range of microbial transfer might lead to high variability in immune phenotype or

332 immune responses. In recently published work, the contributions of individual pathogens on

333 the immune phenotype of cohoused mice were tested, and we found that no single microbe or

334 combination of infections could explain the observed variability in immune activation ${ }^{39}$.

335 Moreover, we found the variability in responses in cohoused mice was not substantially greater

336 than that of SPF mice, which is similar to what has been reported in other publications using

337 cohoused mice $35,36,39$. To press this point, we chose to present data from all experiments

338 conducted rather than show representative experimental repeats, in order to illustrate the full

339 range of responses observed after cohousing.

341 In conclusion, testing airway allergic responses in a mouse model of microbial exposure due to

342 physiological transmission of murine pathogens and commensals has revealed that infectious

343 history does not have a robust and lasting effect on reducing allergic airway responses, unlike

344 acute exposure to microbial products. Despite the long-lasting impacts that microbial exposure

345 through cohousing has on the immune response systemically and in the lungs, it may be that

346 acute triggers of inflammation are the relevant inhibitors of allergic immune responses. These 
347 findings have direct implications for how susceptibility to type-2 immune responses against

348 allergens can be effectively managed. 


\section{Methods}

350 Mice

351 Six- to 8-week old female C57BL/6 (B6) and BALB/CJ mice were purchased from Charles River

352 (via the National Cancer Institute) il5 venus/venus BALB/c mice were kindly provided by Dr. Kiyoshi

353 Takatsu, Toyama University, Toyama, Japan ${ }^{49}$. These mice were bred in-house with B6 mice for

354 one generation to produce $(\mathrm{B} 6 \times \mathrm{BALB} / \mathrm{C})_{\mathrm{F} 1}$ mice heterozygous for the cytokine reporter (called

355 IL-5v F1). Pet store mice were purchased from Twin Cities area pet stores and cohoused with

356 SPF mice as described ${ }^{35}$. Only female mice were used because of the ethics of introducing a

357 new mouse to a cage of adult male mice; males cannot be cohoused as this creates animal

358 welfare concerns due to fighting, aggression, and social defeat. Screening for infectious agents

359 was done as described ${ }^{35}$. Mice were used for experiments between 14 and 17 days or 60 and

360120 days post-cohousing ( $2 \mathrm{wk} \mathrm{CoH}$ and $>2 \mathrm{mo} \mathrm{CoH}$, respectively in figures). Upon euthanasia, a

361 small number of cohoused mice were found to have overt lung pathology, which did not

362 correlate with the appearance or behavior of the live mice. Pathology consisted of pale white or

363 gray tissue, mottled in appearance and of a tougher consistency than healthy lungs, making up

364 at least an estimated $25 \%$ of the outer surface of the lungs, up to $100 \%$. We did not find this

365 pathology significantly impacted the results of the studies and were left in the analysis. Animals

366 were maintained in A-BSL3 under specific pathogen-free, or germ free conditions at the

367 University of Minnesota. All experimental procedures were approved by the Institutional

368 Animal Care and Use Committee at the University of Minnesota.

\section{Serum cytokine measurements}


371 SPF mice were bled prior to cohousing with pet store mice, as well as on various days during the

372 60-day cohousing conditioning period. Serum cytokines and chemokines were quantitated

373 according to manufacturer instructions using a ProcartaPlex custom 7-plex panel (CXCL10, IL-

$3741 \beta$, IL-4, IL-6, IL-10, IFN- $\gamma$, and TNF- $\alpha$; Invitrogen) using a Luminex 200 with Bio-plex Manager

375 Software 5.0. Samples with a reading below the limit of detection were assigned a

376 concentration of $0 \mathrm{pg} / \mathrm{ml}$.

377

378 Airway administration of $A$. alternata extract, HpARI, rIL-33, and poly(I:C)

379 Alternaria alternata extract (Alt, Greer Laboratories, Lenoir, NC, $100 \mu \mathrm{g}$ in $40 \mu \mathrm{l}$ PBS for B6 and

380 F1 mice, $50 \mu \mathrm{g}$ in $50 \mu \mathrm{l}$ PBS for BALB/c mice), recombinant IL-33 (R\&D Systems, Minneapolis,

$381 \mathrm{MN}, 200 \mathrm{ng} /$ dose in $40 \mu \mathrm{l}$ ), or PBS were administered intranasally (i.n.) once or three times

382 (days 0,2 , and 4) to mice anesthetized with isoflurane. In some experiments, recombinant

$383 \mathrm{HpARI}^{30}(10 \mu \mathrm{g})$ was mixed with the Alt. In some experiments mice were pre-treated with i.n.

384 poly(I:C) (Invivogen, San Diego, CA, 50 $\mu$ in $40 \mu$ PBS) 24 hours before Alt treatment.

385

386 Lung and BAL cytokine measurements

387 At the indicated time points, mice were killed by an overdose of isoflurane. The trachea was

388 cannulated, and the lungs were lavaged two times with HBSS $(2 \times 0.5 \mathrm{ml})$. Lungs were then

389 collected. The BAL fluid and lungs were stored at $-80^{\circ} \mathrm{C}$ for cytokine assays. Lungs were

390 homogenized with a glass dounce in $1.0 \mathrm{ml}$ PBS with Halt protease and phosphatase inhibitor

391 (Thermo Scientific). The homogenates were centrifuged at $10,000 \mathrm{rpm}$ at $4^{\circ} \mathrm{C}$ for $15 \mathrm{~min}$, and

392 the protein concentrations in the supernatant were quantified with the BCA Protein Assay kit 
393 (Thermo Scientific). The levels of IL-5, IL-13, and IL-33 in lung homogenate and BAL

394 supernatants were measured by Quantikine ELISA kits (R\&D systems) per manufacturer

395 instructions.

$397 \quad$ Flow cytometry

398 At the indicated time points, mice were killed by an overdose of isoflurane. Lymphocytes were 399 isolated from lungs as previously described ${ }^{50}$, except no Percoll step was performed. Instead, 400 after the GentleMax tissue dissociation step, red blood cells were lysed by incubating for 10 401 minutes in $10 \mathrm{ml} \mathrm{ACK}$ buffer and washed in $20 \mathrm{ml} \mathrm{RPMI}$ with 3\% FBS. In experiments where 402 Tregs were identified, $50 \mu \mathrm{g}$ of Treg-Protector (anti-ARTC2.2) nanobodies (BioLegend) were 403 injected i.v. 15-30 minutes prior to mouse sacrifice as described (Borges da Silva 2018). Direct 404 ex vivo staining was performed as described previously ${ }^{51}$ with fluorochrome-conjugated 405 antibodies (purchased from BD Biosciences, BioLegend, eBioscience, Cell Signaling Technology,

406 Tonbo or Thermo Fisher Scientific). For discrimination of vascular-associated lymphocytes in 407 non-lymphoid organs, in vivo i.v. injection of FITC, vF450, or BUV605 conjugated CD45 antibody 408 was performed as described ${ }^{52}$. For survival assessment, cells were stained with Live/Dead

409 (Tonbo Biosciences) For detection of intracellular factors surface stained cells were

410 permeabilized, fixed and stained by using the eBioscience Foxp3 staining kit, according to

411 manufacturer instructions. Flow cytometric analysis was performed on LSR Fortessa (BD

412 Biosciences) and data was analyzed using FlowJo software (Treestar).

414 Statistics 
415 Unless noted, measurements were taken from distinct samples. GraphPad Prism was used to 416 determine statistical significance. Student unpaired two-tailed t-test, one-way ANOVA, or two417 way ANOVA with Tukey's multiple comparisons test was used when appropriate. For two-way 418 ANOVA tests, only relevant comparisons were shown (e.g. we did not report SPF PBS vs. >2mo $419 \mathrm{CoH}$ ALT). In Figure 5 when six groups were compared only comparisons that reached statistical 420 significance were reported in order to improve clarity. A $p$ value $<0.05$ was considered 421 statistically significant. 


\section{Acknowledgements}

423 We thank the UMN Flow Cytometry Resource Facility, CFI Dirty Mouse Colony, BSL-3 Program, 424 and UMN Research Animal Resources for support. This work was supported by the NIH (R01

425 HL117823 to H.K.; T32 HL007741 to K.E.B., R35 GM140881 to T.S.G.) and funding by the

426 Minnesota Partnership for Biotechnology and Medical Genomics (\#16.48 to S.C.J. and H.K.) and

427 the University of Minnesota Medical School (AIRP-CP-21 to S.C.J.).

428

429 Author contributions

430 K.E.B. designed and performed the experiments and analyzed and interpreted the data. K.I., 431 M.J.P, D.A.W., R.T., T.A.K., J.X., and T.S.G. performed experiments. H.J.M. provided the HpARI

432 reagent. S.C.J. and H.K. supervised the project. K.E.B. and S.C.J. wrote the manuscript. H.K., 433 T.S.G. and H.J.M. edited the manuscript.

434

435 Competing interests

436 The authors declare no competing interests. 


\section{References}

439 1. Croisant, S. Epidemiology of asthma: prevalence and burden of disease. Adv Exp Med Biol 795, 17-29 (2014).

441

2. Holgate, S.T. Innate and adaptive immune responses in asthma. Nat Med 18, 673-683 (2012).

3. Kubo, M. Innate and adaptive type 2 immunity in lung allergic inflammation. Immunol Rev 278, 162-172 (2017).

4. Vivier, E. et al. Innate Lymphoid Cells: 10 Years On. Cell 174, 1054-1066 (2018).

5. Zhu, J. Thelper 2 (Th2) cell differentiation, type 2 innate lymphoid cell (ILC2) development and regulation of interleukin-4 (IL-4) and IL-13 production. Cytokine 75, 14-24 (2015).

6. Halim, T.Y. et al. Group 2 innate lymphoid cells are critical for the initiation of adaptive T helper 2 cell-mediated allergic lung inflammation. Immunity 40, 425-435 (2014).

7. Halim, T.Y. et al. Group 2 innate lymphoid cells license dendritic cells to potentiate memory TH2 cell responses. Nat Immunol 17, 57-64 (2016).

8. Lambrecht, B.N. \& Hammad, H. The immunology of the allergy epidemic and the hygiene hypothesis. Nat Immunol 18, 1076-1083 (2017).

9. Pivniouk, V., Gimenes Junior, J.A., Honeker, L.K. \& Vercelli, D. The role of innate immunity in asthma development and protection: Lessons from the environment. Clin Exp Allergy 50, 282-290 (2020).

10. Kaplan, B.A., Mascie-Taylor, C.G. \& Boldsen, J. Birth order and health status in a British national sample. J Biosoc Sci 24, 25-33 (1992).

11. Ball, T.M. et al. Siblings, day-care attendance, and the risk of asthma and wheezing during childhood. N Engl J Med 343, 538-543 (2000).

12. Kramer, U., Heinrich, J., Wjst, M. \& Wichmann, H.E. Age of entry to day nursery and allergy in later childhood. Lancet 353, 450-454 (1999).

13. Riedler, J., Eder, W., Oberfeld, G. \& Schreuer, M. Austrian children living on a farm have less hay fever, asthma and allergic sensitization. Clin Exp Allergy 30, 194-200 (2000).

14. Riedler, J. et al. Exposure to farming in early life and development of asthma and allergy: a cross-sectional survey. Lancet 358, 1129-1133 (2001). 
15. Ege, M.J. et al. Exposure to environmental microorganisms and childhood asthma. $N$ Engl J Med 364, 701-709 (2011).

16. Sjögren, Y.M., Jenmalm, M.C., Böttcher, M.F., Björkstén, B. \& Sverremark-Ekström, E. Altered early infant gut microbiota in children developing allergy up to 5 years of age. Clin Exp Allergy 39, 518-526 (2009).

17. Bisgaard, H. et al. Reduced diversity of the intestinal microbiota during infancy is associated with increased risk of allergic disease at school age. J Allergy Clin Immunol 128, 646-652.e641-645 (2011).

18. Stein, M.M. et al. Innate Immunity and Asthma Risk in Amish and Hutterite Farm

19. Marra, F. et al. Antibiotic use in children is associated with increased risk of asthma.

20. van Tilburg Bernardes, E. \& Arrieta, M.C. Hygiene Hypothesis in Asthma Development: Is Hygiene to Blame? Arch Med Res 48, 717-726 (2017).

21. Kline, J.N. et al. Modulation of airway inflammation by CpG oligodeoxynucleotides in a murine model of asthma. J Immunol 160, 2555-2559 (1998).

22. Sur, S. et al. Long term prevention of allergic lung inflammation in a mouse model of asthma by CpG oligodeoxynucleotides. J Immunol 162, 6284-6293 (1999).

23. Sabatel, C. et al. Exposure to Bacterial CpG DNA Protects from Airway Allergic Inflammation by Expanding Regulatory Lung Interstitial Macrophages. Immunity 46, 457473 (2017).

24. Schuijs, M.J. et al. Farm dust and endotoxin protect against allergy through A20 induction in lung epithelial cells. Science 349, 1106-1110 (2015).

25. Tei, R. et al. TLR3-driven IFN- $\beta$ antagonizes STAT5-activating cytokines and suppresses innate type 2 response in the lung. J Allergy Clin Immunol (2021).

26. Pivniouk, V. et al. Airway administration of OM-85, a bacterial lysate, blocks experimental asthma by targeting dendritic cells and the epithelium/IL-33/ILC2 axis. J Allergy Clin Immunol (2021).

27. Eisenbarth, S.C. et al. Lipopolysaccharide-enhanced, toll-like receptor 4-dependent T helper cell type 2 responses to inhaled antigen. J Exp Med 196, 1645-1651 (2002). 
28. Hammad, H. et al. House dust mite allergen induces asthma via Toll-like receptor 4 triggering of airway structural cells. Nat Med 15, 410-416 (2009).

29. McSorley, H.J., Blair, N.F., Smith, K.A., McKenzie, A.N. \& Maizels, R.M. Blockade of IL-33 release and suppression of type 2 innate lymphoid cell responses by helminth secreted products in airway allergy. Mucosal Immunol 7, 1068-1078 (2014).

30. Osbourn, M. et al. HpARI Protein Secreted by a Helminth Parasite Suppresses Interleukin-33. Immunity 47, 739-751.e735 (2017).

31. Machiels, B. et al. A gammaherpesvirus provides protection against allergic asthma by inducing the replacement of resident alveolar macrophages with regulatory monocytes. Nat Immunol 18, 1310-1320 (2017).

32. Molofsky, A.B. et al. Interleukin-33 and Interferon- $\gamma$ Counter-Regulate Group 2 Innate Lymphoid Cell Activation during Immune Perturbation. Immunity 43, 161-174 (2015).

33. Moro, K. et al. Interferon and IL-27 antagonize the function of group 2 innate lymphoid cells and type 2 innate immune responses. Nat Immunol 17, 76-86 (2016).

34. Duerr, C.U. et al. Type I interferon restricts type 2 immunopathology through the regulation of group 2 innate lymphoid cells. Nat Immunol 17, 65-75 (2016).

35. Beura, L.K. et al. Normalizing the environment recapitulates adult human immune traits in laboratory mice. Nature 532, 512-516 (2016).

36. Huggins, M.A. et al. Microbial Exposure Enhances Immunity to Pathogens Recognized by TLR2 but Increases Susceptibility to Cytokine Storm through TLR4 Sensitization. Cell Rep 28, 1729-1743.e1725 (2019).

37. Bartemes, K.R. et al. IL-33-responsive lineage- CD25+ CD44(hi) lymphoid cells mediate innate type 2 immunity and allergic inflammation in the lungs. $J$ Immunol 188, 15031513 (2012).

38. Jabbari, A., Legge, K.L. \& Harty, J.T. T cell conditioning explains early disappearance of the memory CD8 T cell response to infection. J Immunol 177, 3012-3018 (2006).

39. Fiege, J.K. et al. Mice with diverse microbial exposure histories as a model for preclinical vaccine testing. Cell Host Microbe (2021).

40. Oboki, K. et al. IL-33 is a crucial amplifier of innate rather than acquired immunity. Proc Natl Acad Sci U S A 107, 18581-18586 (2010). 
41. Cayrol, C. \& Girard, J.P. IL-33: an alarmin cytokine with crucial roles in innate immunity, inflammation and allergy. Curr Opin Immunol 31, 31-37 (2014).

42. Hamilton, S.E. et al. New Insights into the Immune System Using Dirty Mice. J Immunol 205, 3-11 (2020).

43. Masopust, D., Sivula, C.P. \& Jameson, S.C. Of Mice, Dirty Mice, and Men: Using Mice To Understand Human Immunology. J Immunol 199, 383-388 (2017).

44. Wilson, M.S. et al. Suppression of allergic airway inflammation by helminth-induced regulatory T cells. J Exp Med 202, 1199-1212 (2005).

45. Hartmann, S. et al. Gastrointestinal nematode infection interferes with experimental allergic airway inflammation but not atopic dermatitis. Clin Exp Allergy 39, 1585-1596 (2009).

46. Maizels, R.M. Regulation of immunity and allergy by helminth parasites. Allergy 75, 524534 (2020).

47. Wark, P.A.B., Ramsahai, J.M., Pathinayake, P., Malik, B. \& Bartlett, N.W. Respiratory Viruses and Asthma. Semin Respir Crit Care Med 39, 45-55 (2018).

48. Han, M. et al. Small Animal Models of Respiratory Viral Infection Related to Asthma. Viruses 10 (2018).

49. Ikutani, M. et al. Identification of innate IL-5-producing cells and their role in lung eosinophil regulation and antitumor immunity. J Immunol 188, 703-713 (2012).

50. Steinert, E.M. et al. Quantifying Memory CD8 T Cells Reveals Regionalization of Immunosurveillance. Cell 161, 737-749 (2015).

51. Skon, C.N. et al. Transcriptional downregulation of S1pr1 is required for the establishment of resident memory CD8+ T cells. Nat Immunol 14, 1285-1293 (2013).

52. Anderson, K.G. et al. Intravascular staining for discrimination of vascular and tissue leukocytes. Nat Protoc 9, 209-222 (2014). 

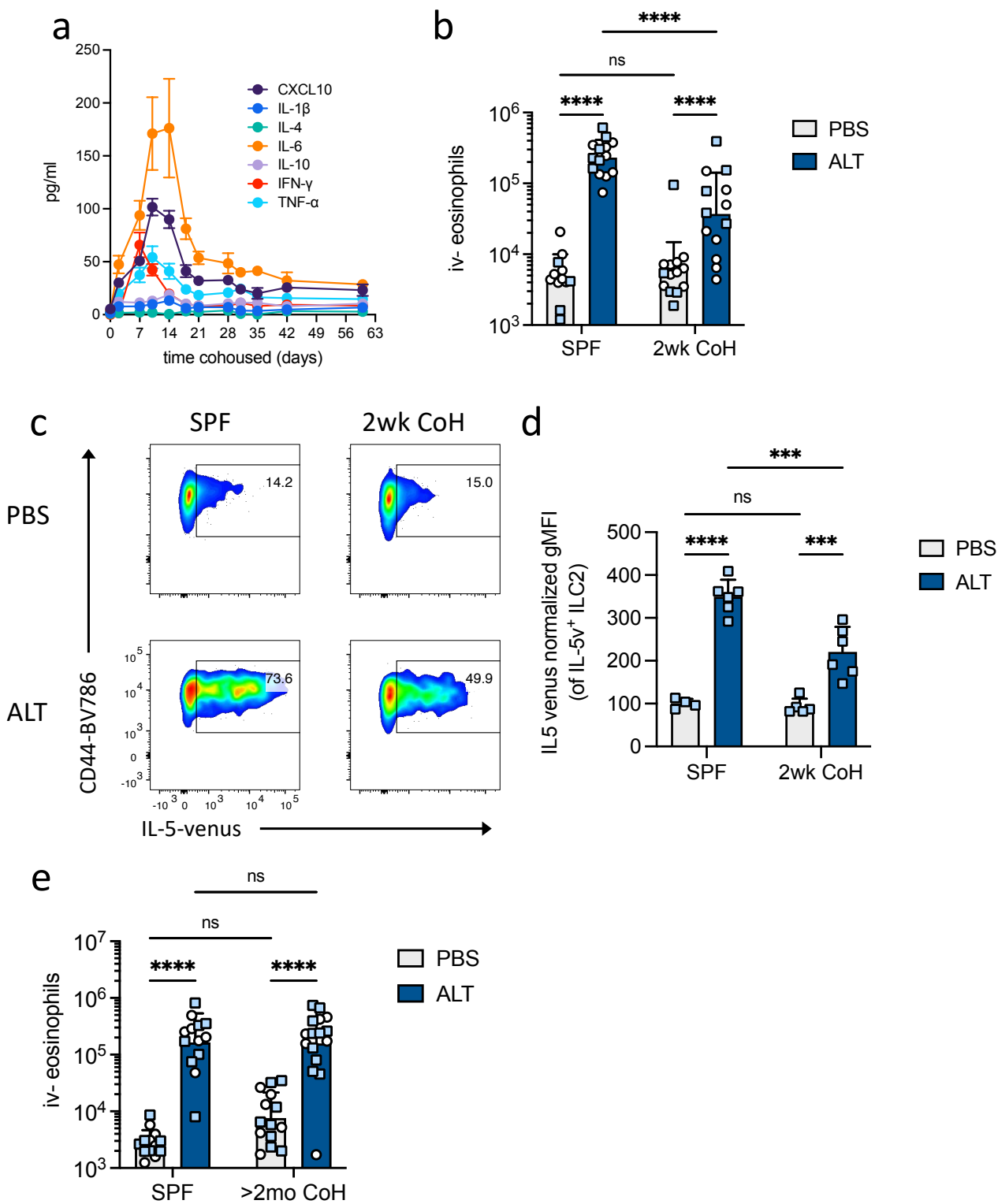

f

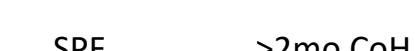

g
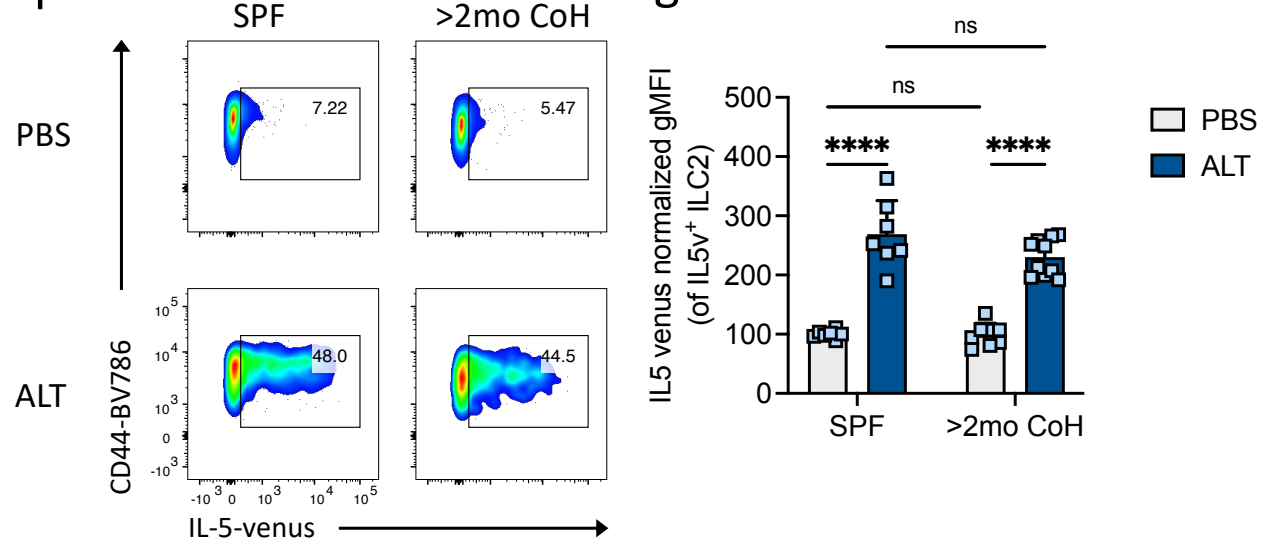
Fig 1. Mice cohoused for two weeks with pet store mice have inhibited eosinophil and ILC2 responses to intranasal $\boldsymbol{A}$. alternata treatment. a, Kinetic changes in serum levels of cytokines and chemokines in C57BL/6 (B6) mice after cohousing with a pet store mouse $(n=8)$. The same

611 cohort of animals were repeatedly measured over time. b-g, Mice were treated with intranasal

612 phosphate buffered saline (PBS) or A. alternata extract in PBS (ALT) and analyzed 24 hours

613 later. Mice were B6 (white circles) or B6xBALB/c IL-5WT/venus (IL-5v F1, light blue squares). Mice

614 were cohoused with pet store mice for approximately two weeks ( 2 wk CoH, b-d) or at least two

615 months (>2mo $\mathrm{CoH}$, e-g), or were age-matched specific pathogen free (SPF) mice. b, e, Number

616 of eosinophils in the lungs and airways (negative for an intravascular CD45 antibody) 24 hours

617 after intranasal treatment. $\mathbf{c}-\mathbf{d}$ and $\mathbf{f}-\mathbf{g}$, IL-5 venus expression within lung ILC2 of IL-5v F1 SPF

618 and 2 wk CoH mice 24 hours after PBS or ALT treatment. c, f, Representative flow plots of IL-5

619 expression in lung ILC2. d, g, Normalized IL-5 venus gMFI of IL-5 venus ${ }^{+}$lung ILC2. b, Pooled

620 from four B6 experiments and two IL-5v F1 experiments ( $n=12-14$ /group). d, Pooled from two

621 IL-5v F1 experiments ( $n=4-6 /$ group). e, Pooled from 3 B6 experiments and four IL-5v F1

622 experiments ( $n=13-16 /$ group). g, Pooled from four IL-5v F1 experiments ( $n=7-10 / g r o u p)$. a,

623 Symbols show mean +/- SD. b, d, e, f, Bar graphs show mean + SD of log-transformed values. $P$

624 values were determined with a 2-way ANOVA with Tukey's multiple comparisons test; ns $p$ >

$6250.05, * * * p<0.001, * * * * p<0.0001$

626 
a

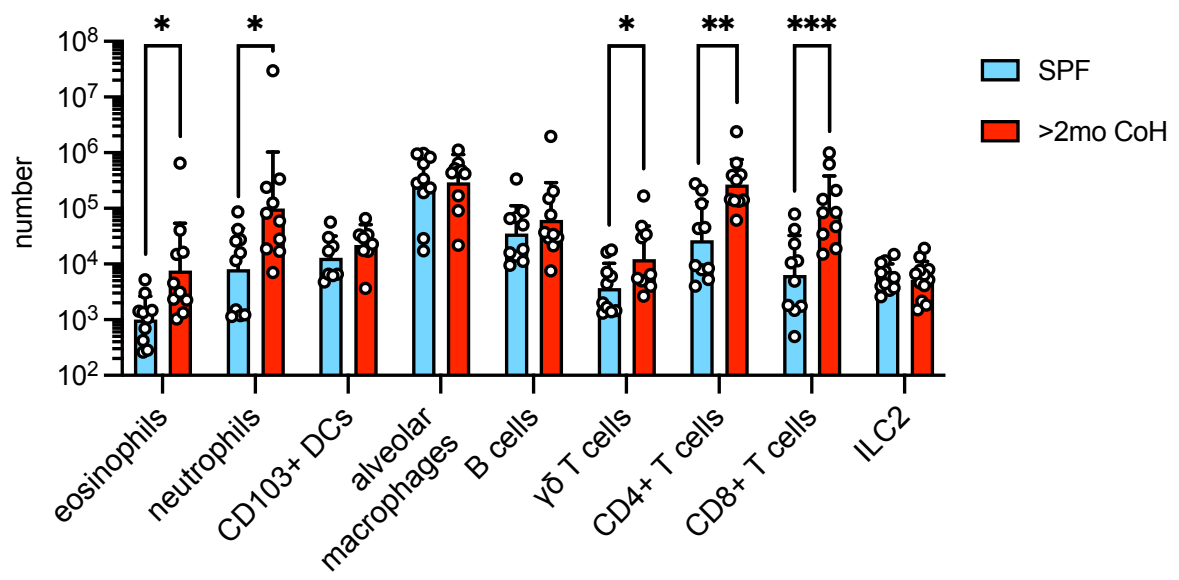

b

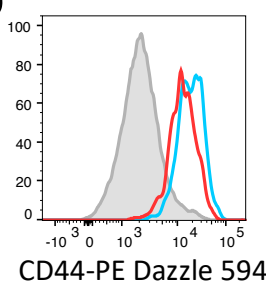

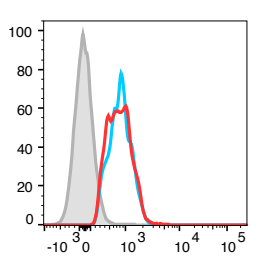

ST2-PE

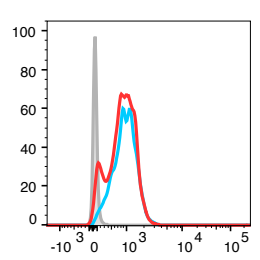

CD25-BV510

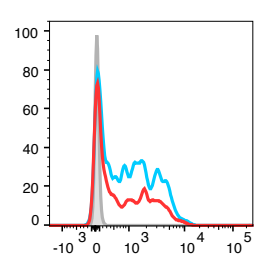

KLRG1-BV421
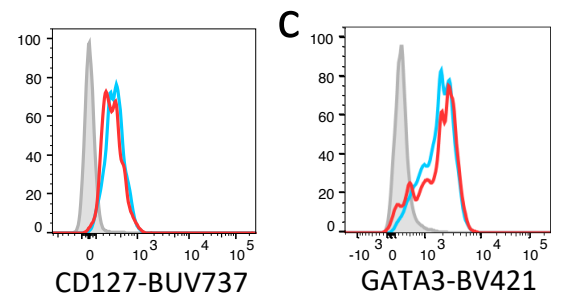

Fig 2. Mouse lung populations are altered by cohousing but ILC2 phenotypes are unchanged.

629 a, Lung immune cell populations of SPF and 2mo CoH B6 mice were identified and quantified by

630 flow cytometry. All enumerated cells were i.v. CD45- except alveolar macrophages, which had

631 an i.v.-intermediate phenotype and were not gated on i.v. status. Pooled from three experiments ( $n=8-12$ /group). Bar graphs show mean + SD of log transformed values. $P$ values were determined with a Student's t-test (two-tailed). Eosinophils had unequal variance and t-

634 test was conducted with Welch's correction; no symbol $p>0.05, * p<0.05, * * p<0.01, * * * p<$

636 (red) mice. I.v. ${ }^{+}$CD19 ${ }^{+}$cells in gray are included as a negative or low expression control. c,

637 Representative intracellular GATA3 expression of lung ILC2 cells. I.v. ${ }^{+}$Lineage $^{+}$cells in gray are included as a negative or low expression control. Representative of three experiments. 

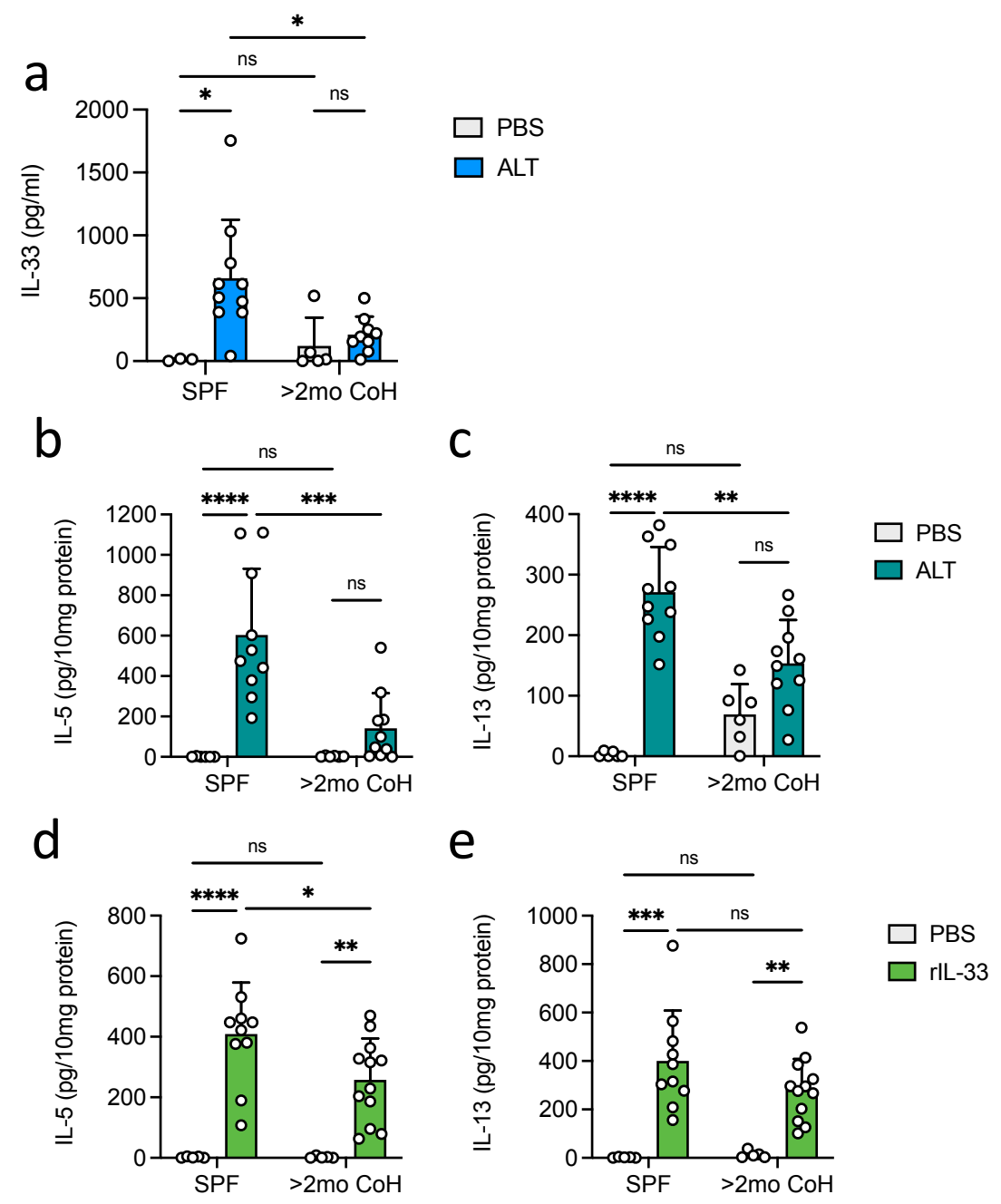

641 Fig 3. Acute IL-33, IL-5 and IL-13 responses after $\boldsymbol{A}$. alternata exposure are reduced in two-

642 month cohoused mice. a, IL-33 in the bronchoalveolar lavage fluid (BALF) of SPF and $>2 \mathrm{mo} \mathrm{CoH}$

643 B6 mice one hour after ALT exposure, detected by ELISA. b-e, ALT (b-c), recombinant IL-33 (rIL-

$64433, d-e)$ or control phosphate buffered saline (PBS) were given intranasally to B6 mice and lungs

645 and BAL were collected 4.5 hours later. IL-5 and IL-13 in the lung homogenates were detected

646 by ELISA and normalized to the amount of total protein in the samples. Bar graphs show mean +

647 SD. $P$ values were determined with 2-way ANOVA with Tukey's multiple comparisons test; ns $p$

$648>0.05,{ }^{*} p<0.05,{ }^{* *} p<0.01,{ }^{* * *} p<0.001, * * * * p<0.0001$. 

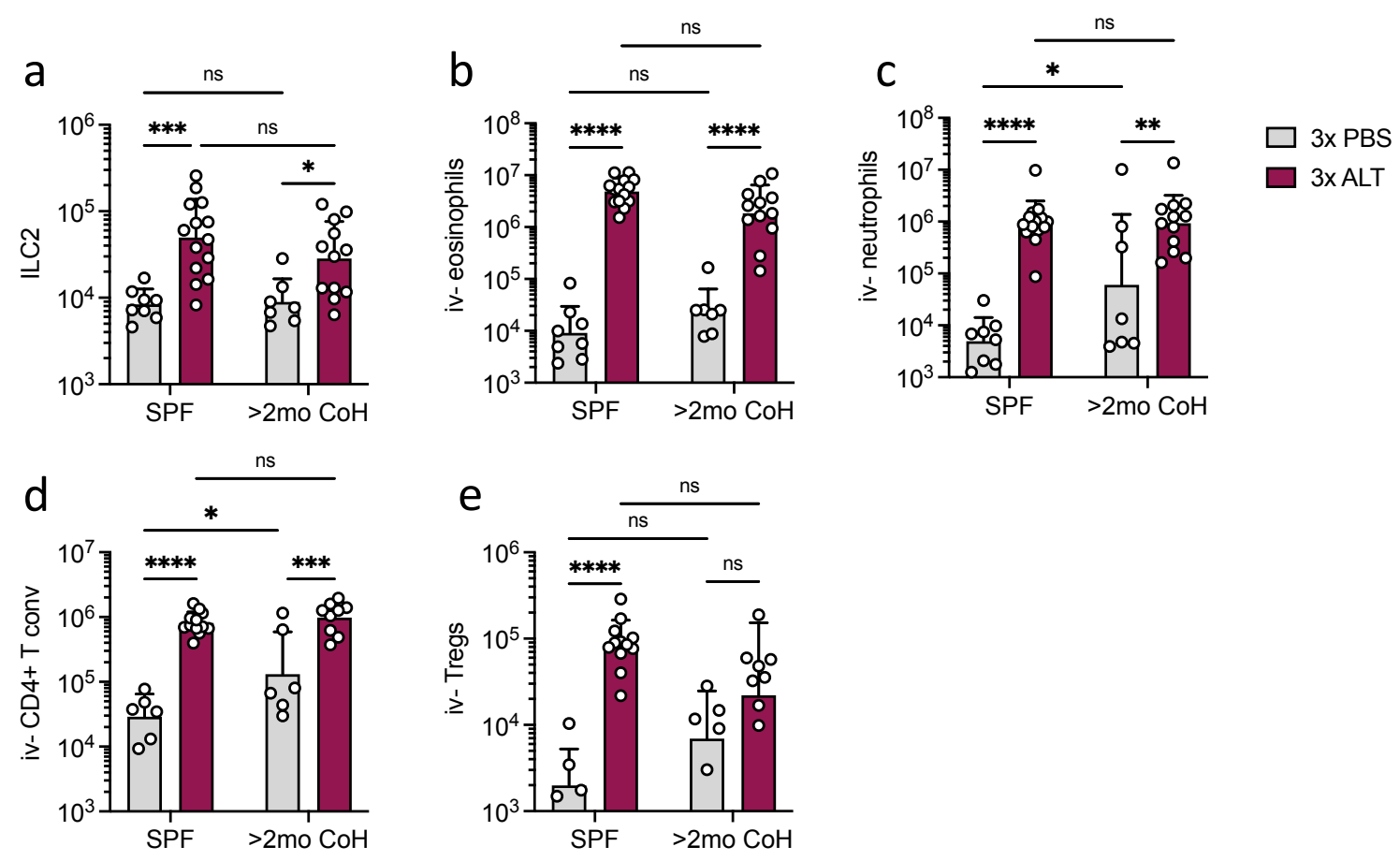

650 Fig 4. Comparable immune infiltration in SPF and two-month cohoused mice after repeated

651 A. alternata exposure. SPF and $>2 \mathrm{mo} \mathrm{CoH} \mathrm{B6}$ mice were treated on days 0,2 , and 4 with 652 intranasal PBS or ALT and on day 5 lungs were harvested for flow cytometric analysis. All

653 enumerated cells were i.v. CD45- a, ILC2, b, eosinophils, c, neutrophils, d, CD4 ${ }^{+}$conventional T

654 cells (Foxp3 $\left.{ }^{\text {neg }}\right)$, and e, Tregs (Foxp3 $3^{+}$CD4 $^{+}$T cells). Pooled from 3-4 experiments $(n=4-$

655 14/group). Bar graphs show mean + SD of log transformed values. $P$ values were determined

656 with two-way ANOVA with Tukey's multiple comparisons test; ns $p>0.05, * p<0.05, * * p<$

$657 \quad 0.01, * * * p<0.001, * * * * p<0.0001$. 

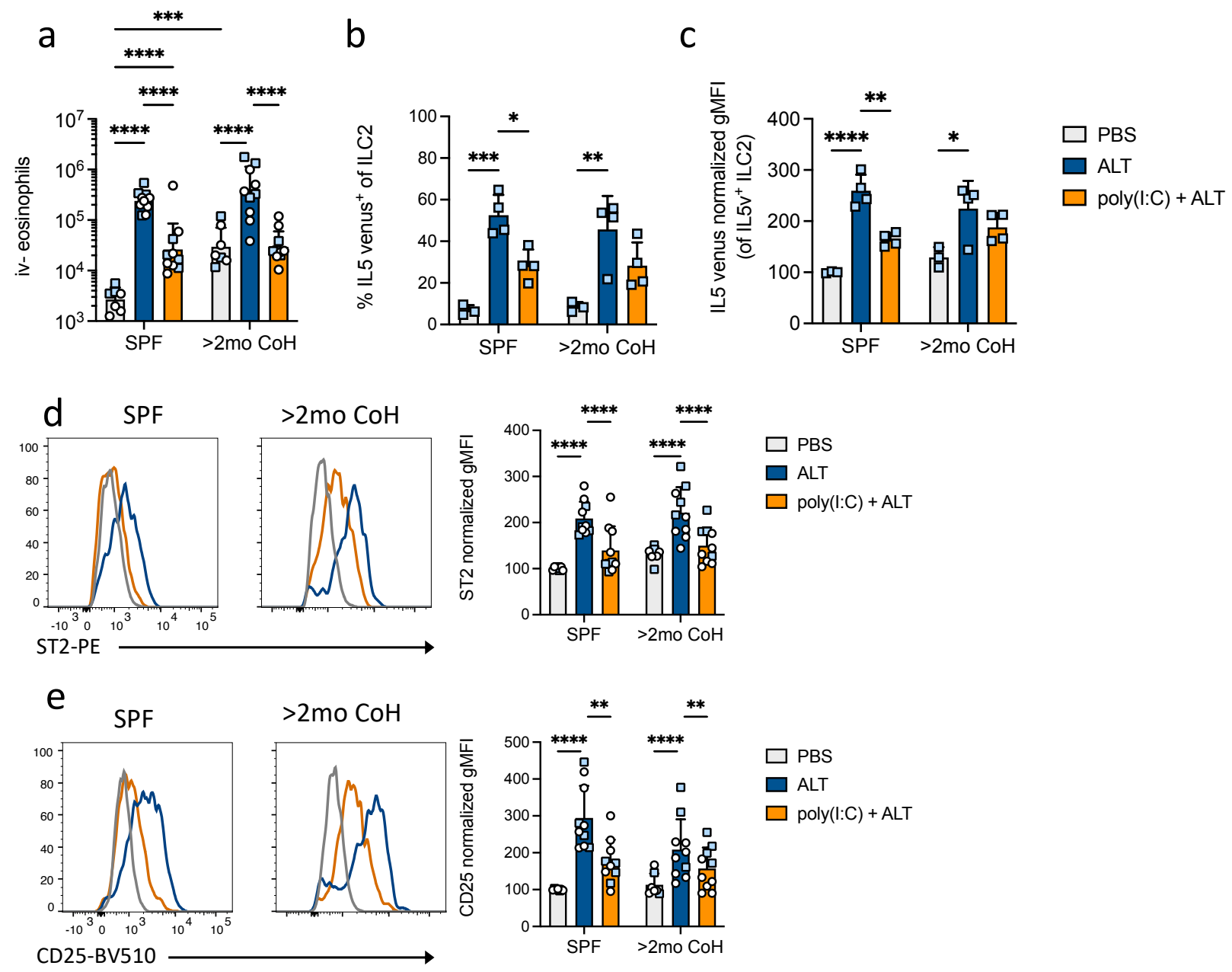

Fig 5. Poly(I:C) suppresses ILC2 and eosinophil responses to ALT in SPF and two-month

cohoused mice. SPF or >2mo CoH B6 (white circles) or IL-5v F1 (light blue squares) mice were

662 treated with intranasal poly $(\mathrm{I}: \mathrm{C})$ or left untreated 24 hours before intranasal PBS or ALT and

663 analyzed by flow cytometry 24 hours later. a, Number of lung i.v. CD45 eosinophils. Bar graph

664 shows mean + SD of log transformed values. b, Percent of lung ILC2 expressing IL-5 venus. c,

665 Normalized gMFI of IL-5 venus, gated on IL-5 venus ${ }^{+}$lung ILC2. d, Representative histograms

666 and summary data of ST2 expression on lung ILC2. e, Representative histograms and summary

667 data of CD25 expression on lung ILC2. a, d-e, Pooled from two B6 and two IL-5v F1 experiments

668 ( $n=7-10$ group). b-c, Pooled from two IL-5v F1 experiments ( $n=3-4 /$ group). b, c, d, e, Bar 
669 graphs show mean + SD. P values were determined with two-way ANOVA with Tukey's multiple 670 comparisons test; no symbol $p>0.05, * p<0.05, * * p<0.01, * * * p<0.001, * * * * p<0.0001$. 


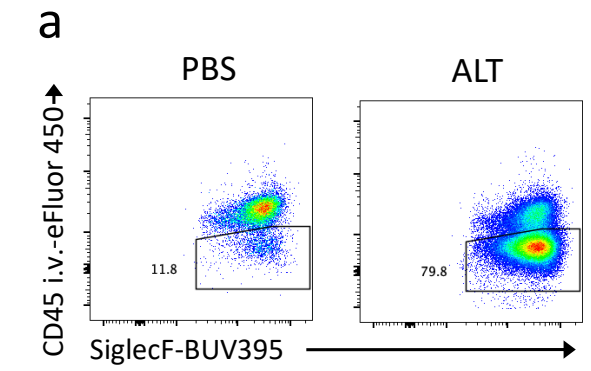

C
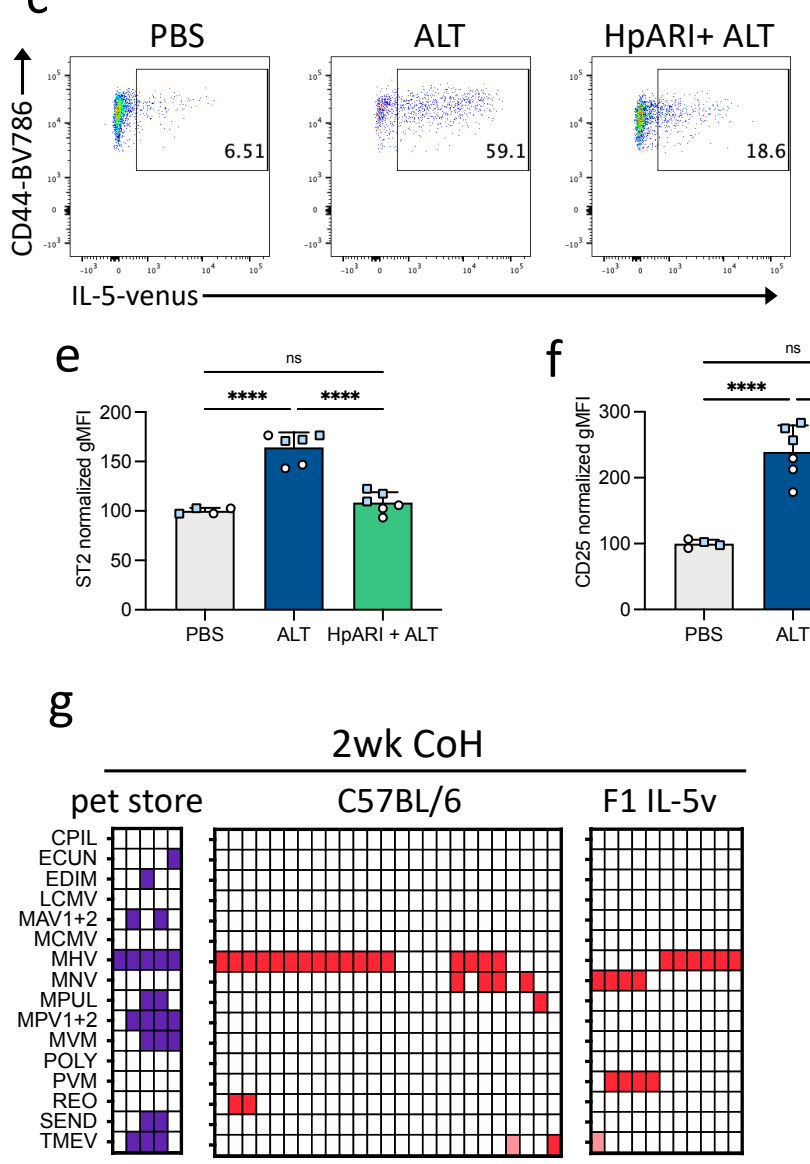

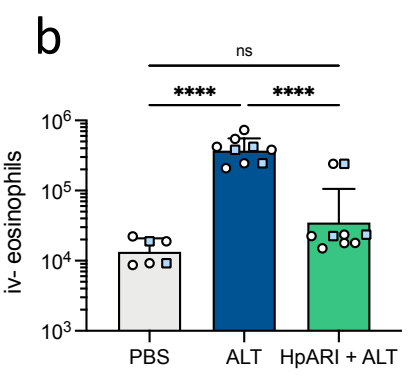

d
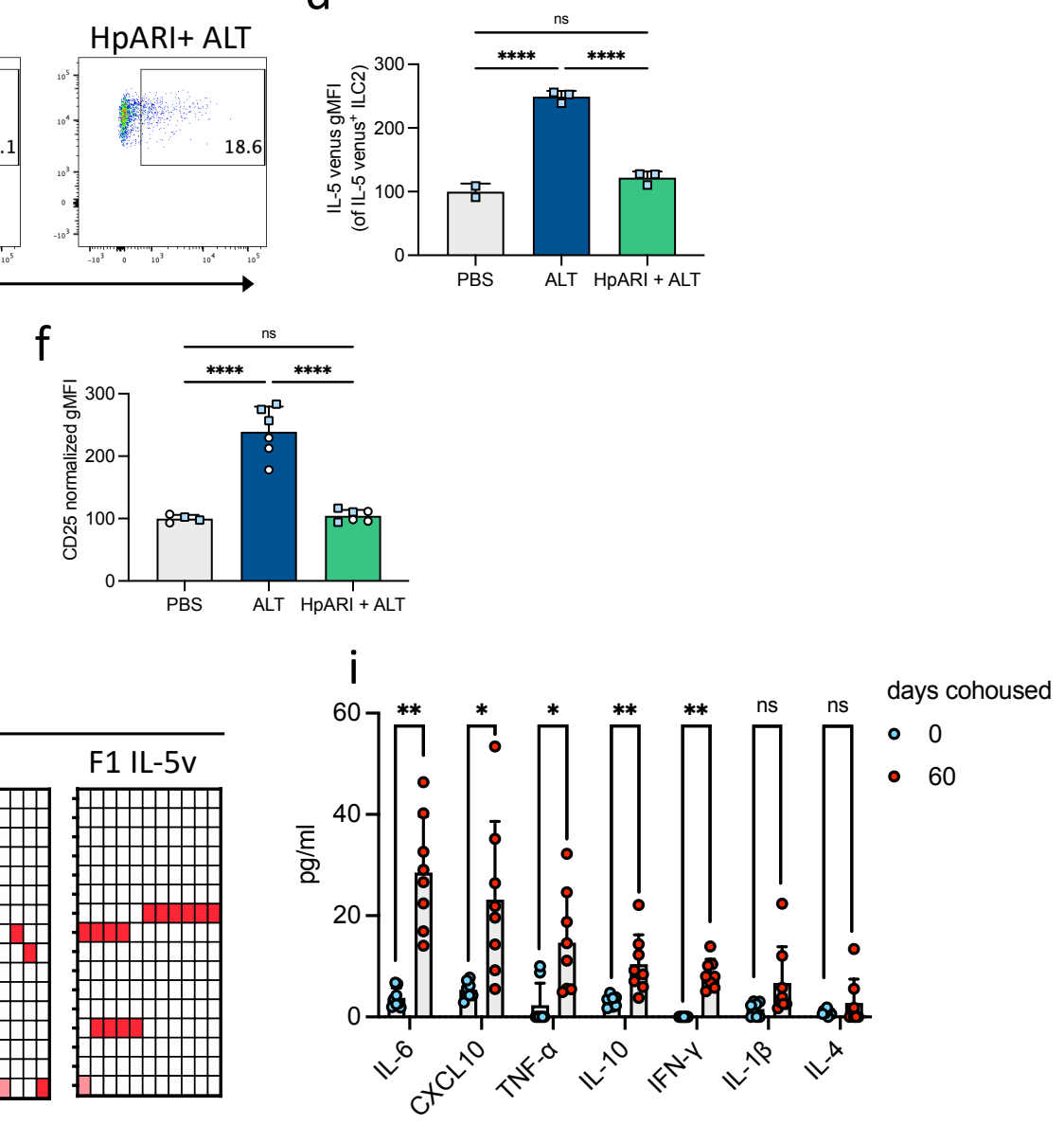

h

$>2 \mathrm{mo} \mathrm{CoH}$

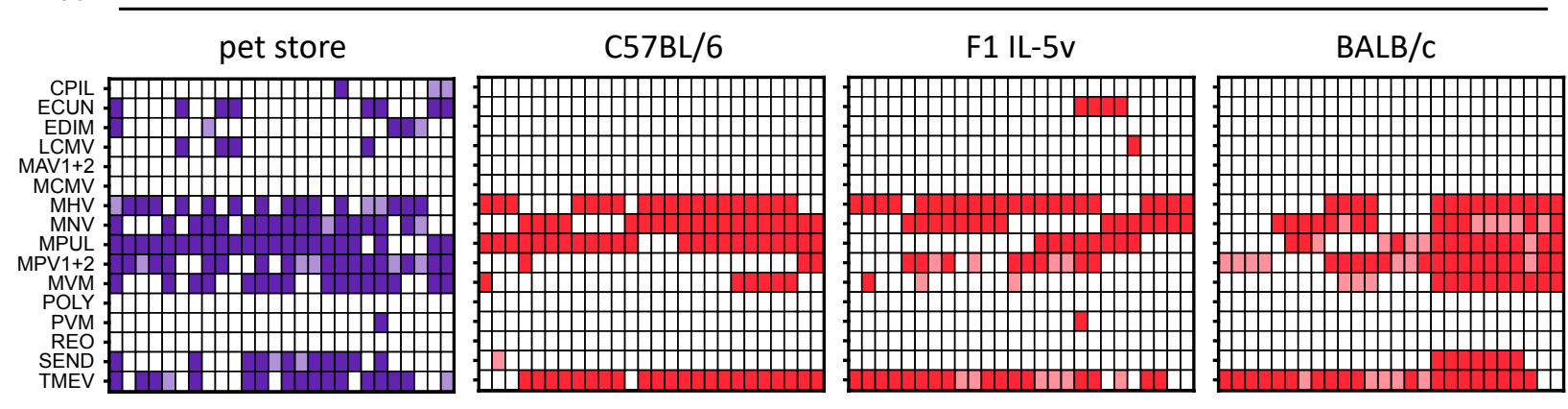


672 Fig S1. A. alternata response is susceptible to inhibition by microbial factors. a, Eosinophils

$673\left(\mathrm{CD}_{4} 5^{+}\right.$SiglecF $\mathrm{CD}^{+} 11 \mathrm{~b}^{+} \mathrm{CD} 11 \mathrm{c}^{-}$cells) were identified by being in the lung parenchyma or

674 airways by being unlabeled by a fluorescently tagged anti-CD45 antibody that was injected

675 intravenously three minutes before euthanasia. Shown are SPF C57BL/6 (B6) mice treated 24

676 hours prior with intranasal PBS or A. alternata (ALT). b, Number of eosinophils in the lungs and

677 airways (negative for an intravascular CD45 antibody) 24 hours after intranasal treatment with

678 PBS, ALT, or ALT co-administered with HpARI. Mice were B6 (white circles) or B6xBALB/c IL-

$6795^{\text {WT/venus }}$ (IL-5v F1, light blue squares). Bar graph shows mean + SD of log transformed values. c,

680 Representative flow plots of IL-5 venus expression in IL-5v F1 lung ILC2. d, Normalized IL-5

681 venus gMFI of IL-5 venus ${ }^{+}$lung ILC2. e-f, Normalized ST2 (e) and CD25 (f) of B6 and IL-5v F1

682 mice. $\mathbf{b}$ and $\mathbf{e - f}$, Pooled from one B6 and one IL-5v F1 experiment ( $n=5-9 /$ group). $\mathbf{d}$, Data from

683 one experiment in IL-5v F1 mice ( $n=2-3$ /group). Bar graphs show mean + SD. $P$ values were

684 determined with one-way ANOVA with Tukey's multiple comparisons test; ns $p>0.05, * * * * p<$

685 0.0001. $\mathbf{g}, \mathbf{h}$, Blood samples were collected and tested for antibodies against common murine

686 pathogens. Each column represents an animal. Each row indicates a pathogen. Filled boxes

687 indicate positive results, lighter shaded boxes indicate equivocal (weak positive) results. Empty

688 boxes indicate negative results. g, Serology results from representative pet store, cohoused B6

689 and F1 IL-5v mice cohoused for approximately two weeks at the time of blood collection. $\mathbf{h}$,

690 Serology results from representative pet store and cohoused B6, F1 IL-5v, and BALB/c mice

691 cohoused for at least two months at the time of blood collection ( $n=26 /$ group). CPIL $=$

692 Clostridium piliforme, ECUN = Encephalitozoon cuniculi, EDIM = rotavirus, LCMV = lymphocytic

693 choriomeningitis virus, MAV1+2 = mouse adenovirus 1 and 2, MCMV = murine cytomegalovirus, 
$694 \mathrm{MHV}=$ mouse hepatitis virus, $\mathrm{MNV}=$ murine norovirus, $\mathrm{MPUL}=$ Mycoplasma pulmonis,

$695 \mathrm{MPV} 1+2=$ mouse parvovirus type 1 and type $2, \mathrm{MVM}=$ minute virus of mice, $\mathrm{POLY}=$ polyoma

696 virus, $\mathrm{PVM}=$ pneumonia virus of mice, $\mathrm{REO}=$ reovirus, SEND = murine respirovirus (Sendai

697 virus), TCMV = GDVII Theiler's murine encephalomyelitis virus. i, Serum cytokine/chemokine

698 levels of B6 mice days 0 and 60 after cohousing with a pet store mouse, $(n=8)$. These are from

699 the same data in figure 1a. Bar graphs show mean + SD. $P$ values were determined with a

700 Wilcoxon matched-pairs signed rank test; ns $p>0.05, * p<0.05,{ }^{* *} p<0.01$. 

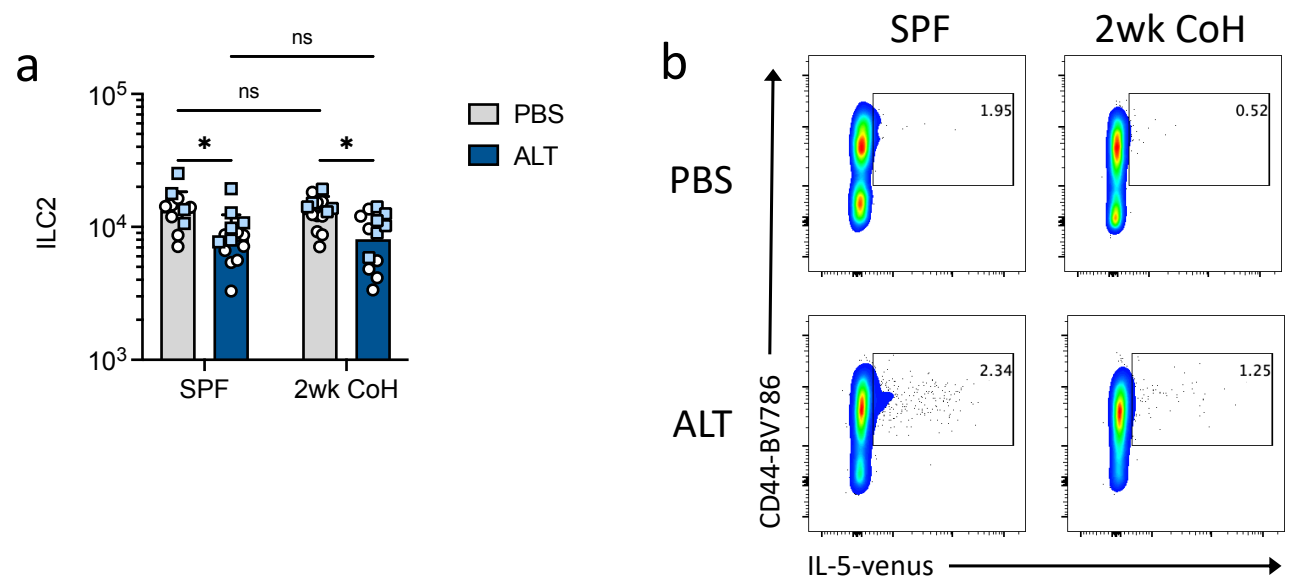

701

C

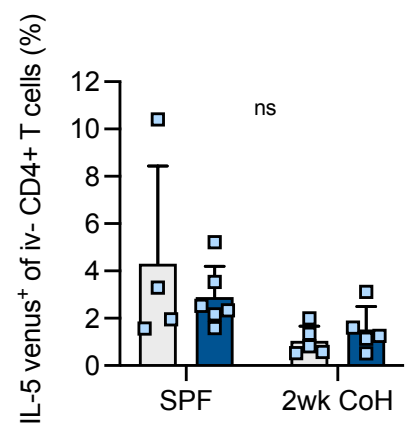

d

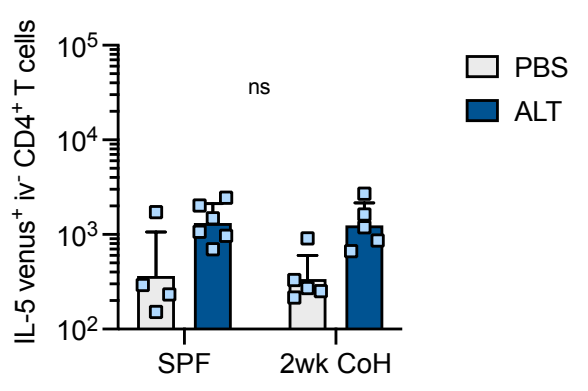

702 Fig S2. ILC2 numbers and CD4+ T cell data from two-week cohoused mice.

703 Flow analysis 24 hours after intranasal PBS or ALT treatment. Mice were B6 (white circles) or IL-

704 5v F1 (light blue squares) and were cohoused with pet store mice for approximately two weeks

705 or were age-matched SPF controls. a, Number of lung ILC2. b-c, IL-5 venus expression within

706 lung (i.v. CD45') CD4 ${ }^{+}$T cells of IL-5v F1 SPF and 2 wk CoH mice 24 hours after PBS or ALT

707 treatment. b, Representative flow plots of IL-5 venus expression in lung $\mathrm{CD} 4^{+} \mathrm{T}$ cells. c, Percent

$708 \mathrm{IL}^{-5}$ venus $^{+}$and $\mathbf{g}$, normalized IL-5 venus gMFI of IL-5 venus ${ }^{+}$lung CD4 ${ }^{+}$T cells. a, Pooled from

709 four B6 experiments and two IL-5v F1 experiments, $n=12-14$ per group. b-d, Pooled from two

710 IL-5v F1 experiments, $n=4-6$ per group. Bar graphs show mean + SD. $P$ values were determined

711 with a two-way ANOVA with Tukey's multiple comparisons test; ns $p>0.05, * p<0.05$. 
a Gating neutrophils, CD103+ DCs, eosinophils, alveolar macrophages (untreated cohoused mouse)
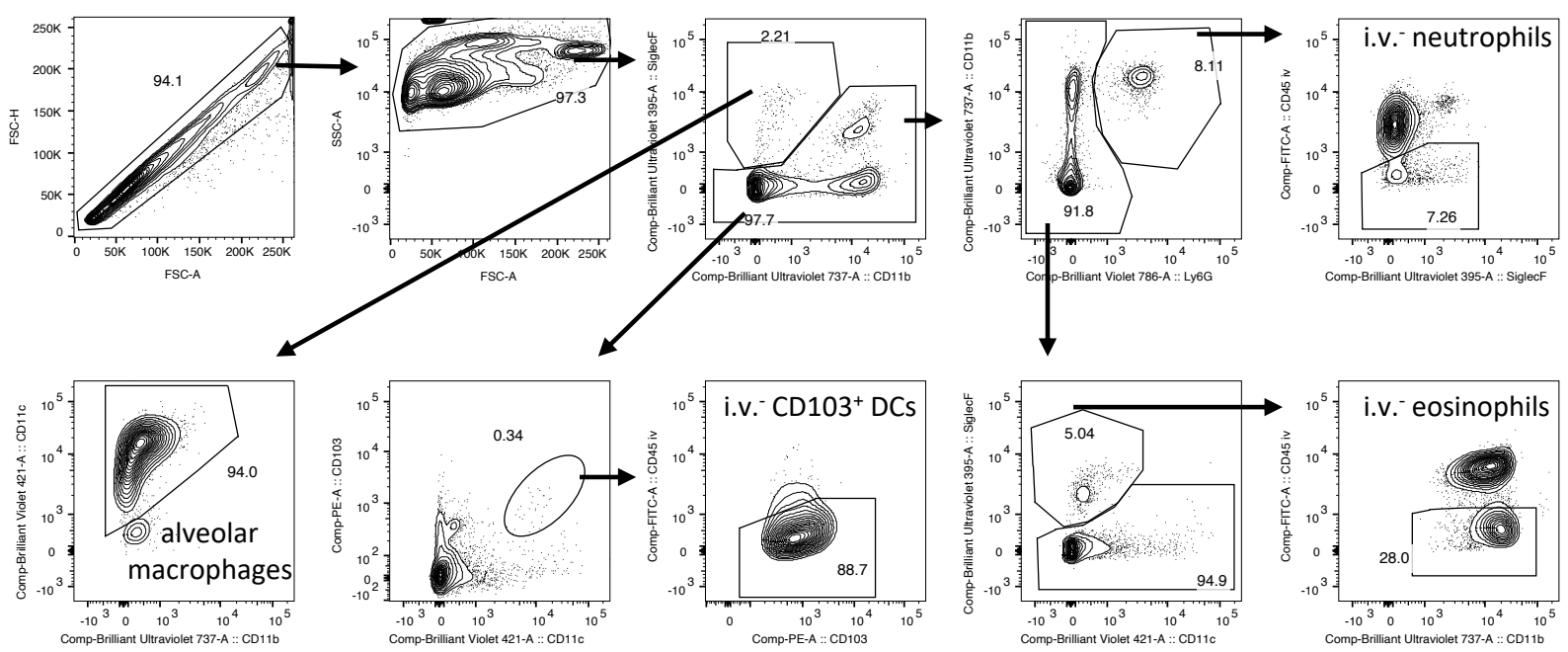

b Gating $\gamma \delta T$ cells, $C D 4^{+} T$ cells, $C D 8^{+} T$ cells, NK cells (untreated cohoused mouse)

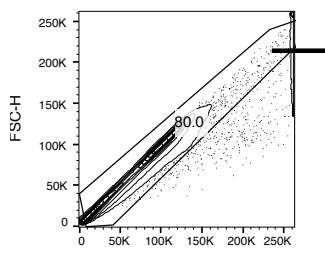

FSC-A

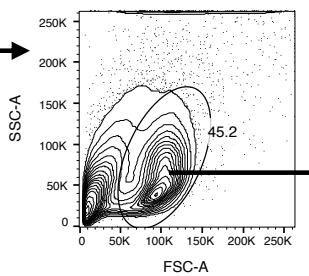

FSC-A
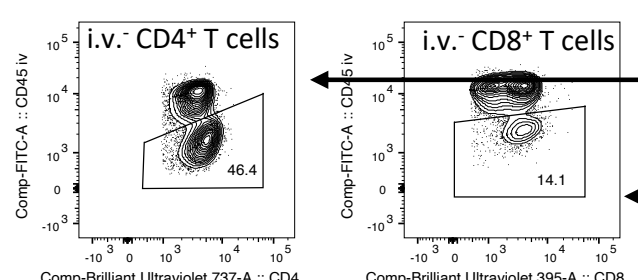

Comp-Brilliant Ultraviolet $395-\mathrm{A}: \because 0^{10}$
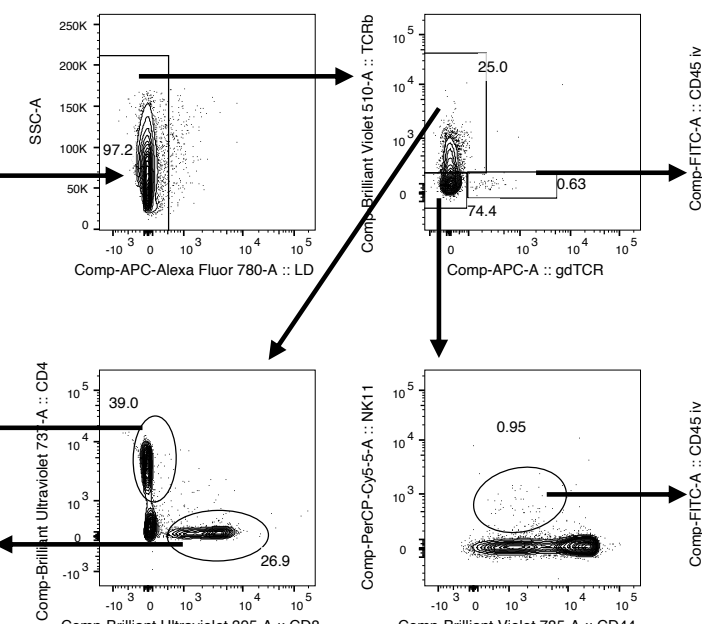

Comp-Brilliant Violet 785-A : CD44
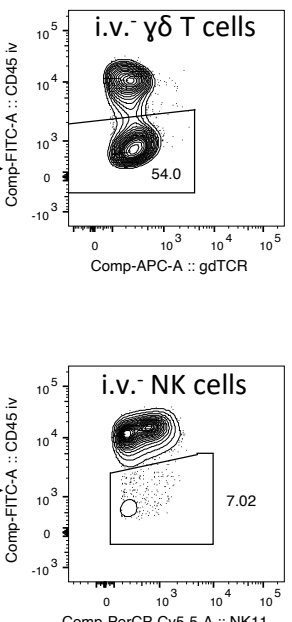

C Gating ILC2, B cells (untreated cohoused mouse), first gated on singlets, lymphocytes, live cells
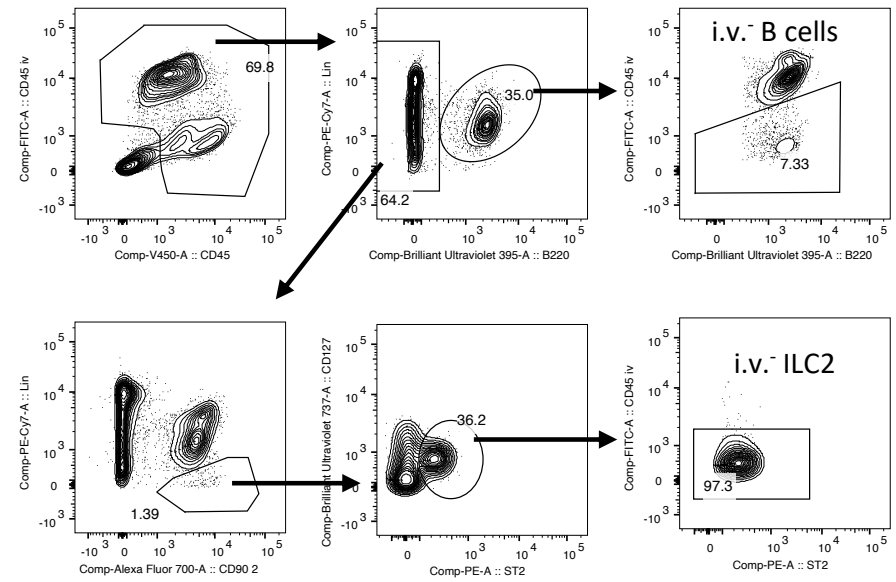
714 Fig S3. Gating strategy for flow cytometry. Example flow cytometry gating used to identify (a)

715 alveolar macrophages and intravenous negative neutrophils, CD103+ dendritic cells,

716 eosinophils, (b) y $\delta$ T cells, $C D 4^{+} T$ cells, $C D 8^{+} T$ cells, NK cells, (c) ILC2 and B cells. The example

717 plots are from $>2 \mathrm{mo} \mathrm{CoH}$ B6 untreated mice as in Figure 2. 

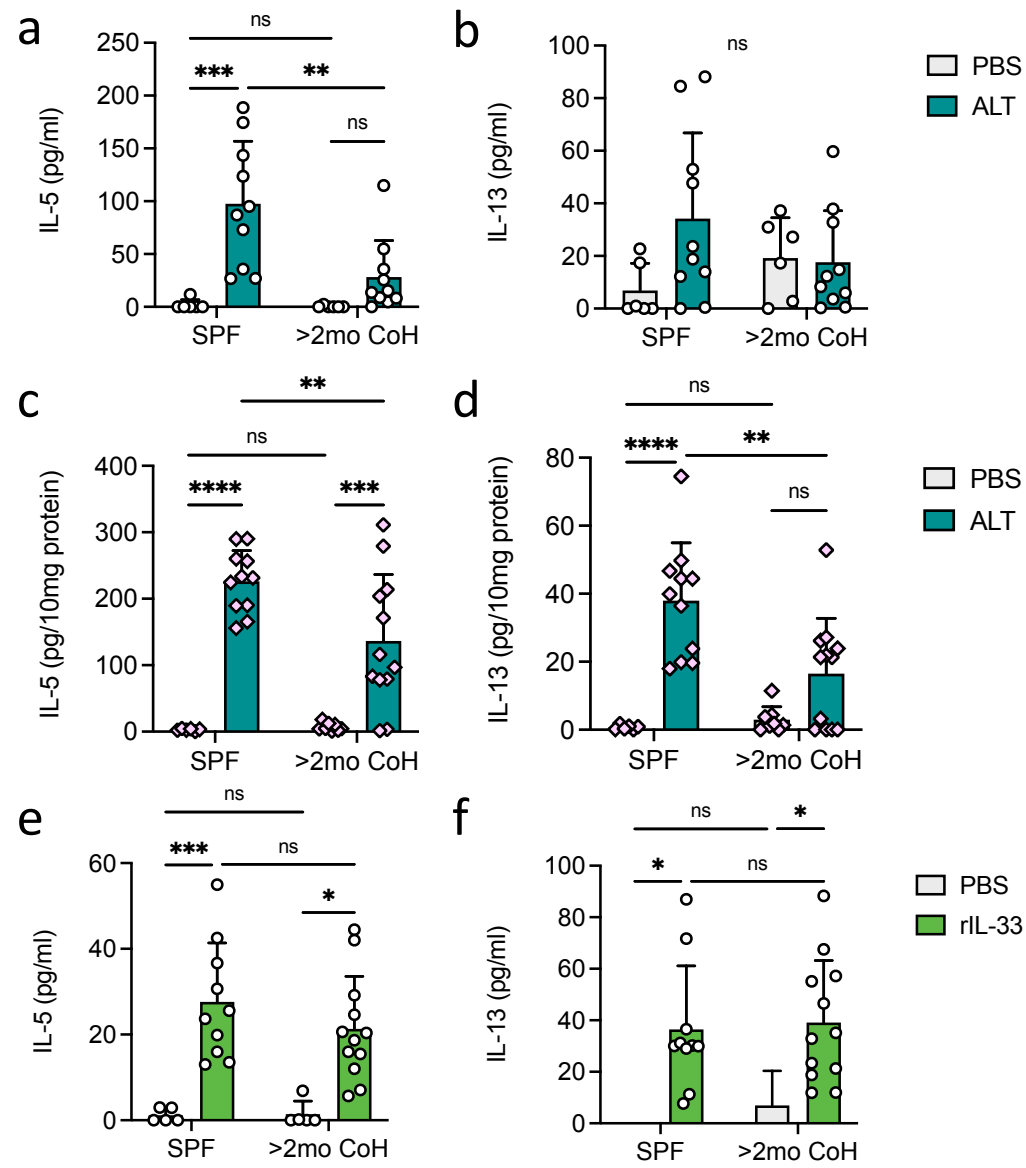

Fig S4. BALF and lung cytokine measurements.

720 a-b, e-f, B6 mice (white circles) or c-d, BALB/c mice (pink diamonds) were cohoused with pet

721 store mice for at least two months (>2mo $\mathrm{CoH})$ or were age-matched SPF controls. ALT (a-d),

722 recombinant IL-33 (rIL-33, e-f) or control PBS were given intranasally to mice and lungs and

723 bronchoalveolar lavage fluid (BALF) were collected 4.5 hours later. IL-5 and IL-13 in the lung

724 homogenates (c-d) and BALF (a-b, e-f) were detected by ELISA and lung concentrations were

725 normalized to the amount of total protein in the samples. Bar graphs show mean + SD. $P$ values

726 were determined with a 2-way ANOVA with Tukey's multiple comparisons test; ns $p>0.05,{ }^{*} p$

$727<0.05, * * p<0.01, * * * p<0.001, * * * * p<0.0001$. 